\title{
JESSE MARTYN [EX]TRACTION EARTH: The New Frontier
}


JESSE MARTYN [EX]TRACTION EARTH: The New Frontier

\section{Artist Statement}

New avenues of interconnectivity born from global crisis foster a return to the land and a decentralization of Earth's population. Climate-induced northern migration sparks boundary expansion beyond our perceivable atmosphere, as human-made infrastructure grapples with the natural environment. Territories of [ex] traction fuel the lifeblood of the 21st century energy-scape.

\section{Project Description}

The site for this investigation is Canada's carbon corridor, spanning from Calgary to Edmonton, Grand Prairie to the remote north. A land scarred by resource exploitation is re-envisioned in a time after oil, in the not so distant future. Territories of extraction are rejuvenated as a renewable energy corridor. A super energy grid is laid across the landscape, linking major urban nodes by coupling the natural environment with infrastructure. Field's within the super grid are dedicated to agricultural production, pollination, and wildlife incubators. These feed natural ecosystems while simultaneously fueling a network of high-speed trains that extend to the arctic circle, where new trade routes open up due to receding ice and warming climates.

A continuous mixed-use bridge structure links people to major hubs as a path for production. Human life is elevated so wildlife may roam freely on the land - reducing harmful impacts to natural ecosystems. Pipelines become long range carbon sequestering systems that distribute green energy to cities, landscapes, wildlife, and insects. Energy beacons are sprinkled across the land to assist birds with natural migration patterns while also acting as habitats for bees.

This project taps into the imperative of clean energy as a catalyst to support humans, wildlife, and insects through closed-loop systems of energy production that prioritize the harnessing of natural resources with minimal environmental impacts. This work investigates the designer's ability to act as a mediator, highlighting the need to consider cycles and transformations. As Fumihiko Maki declared in 1964, "We must now see our urban society as a dynamic field of interrelated forces. It is a set of mutually independent variables in a rapidly expanding infinite series. Any order introduced within the pattern of forces contributes to a state of dynamic equilibrium - an equilibrium which will change in character as time passes." 


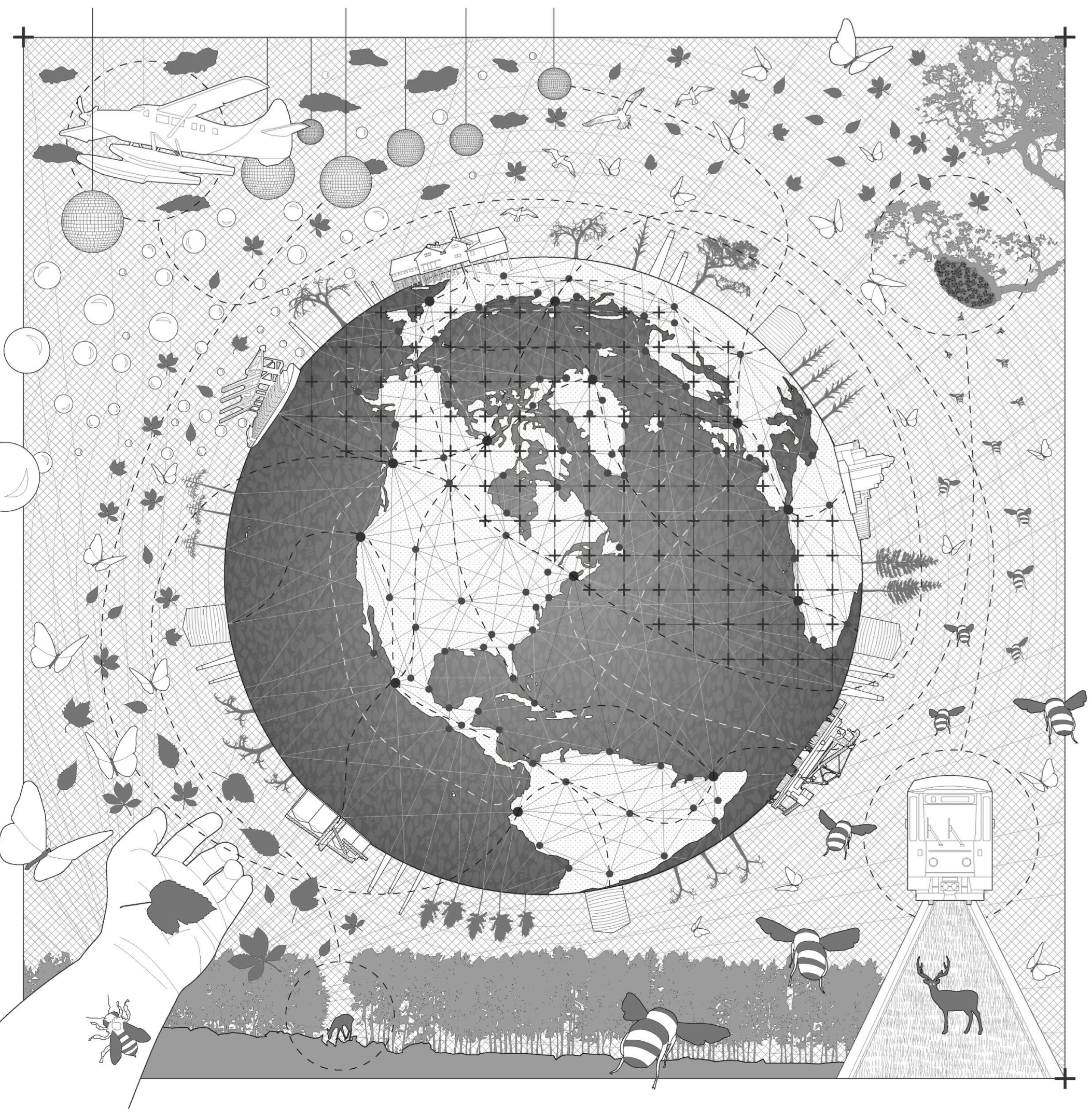




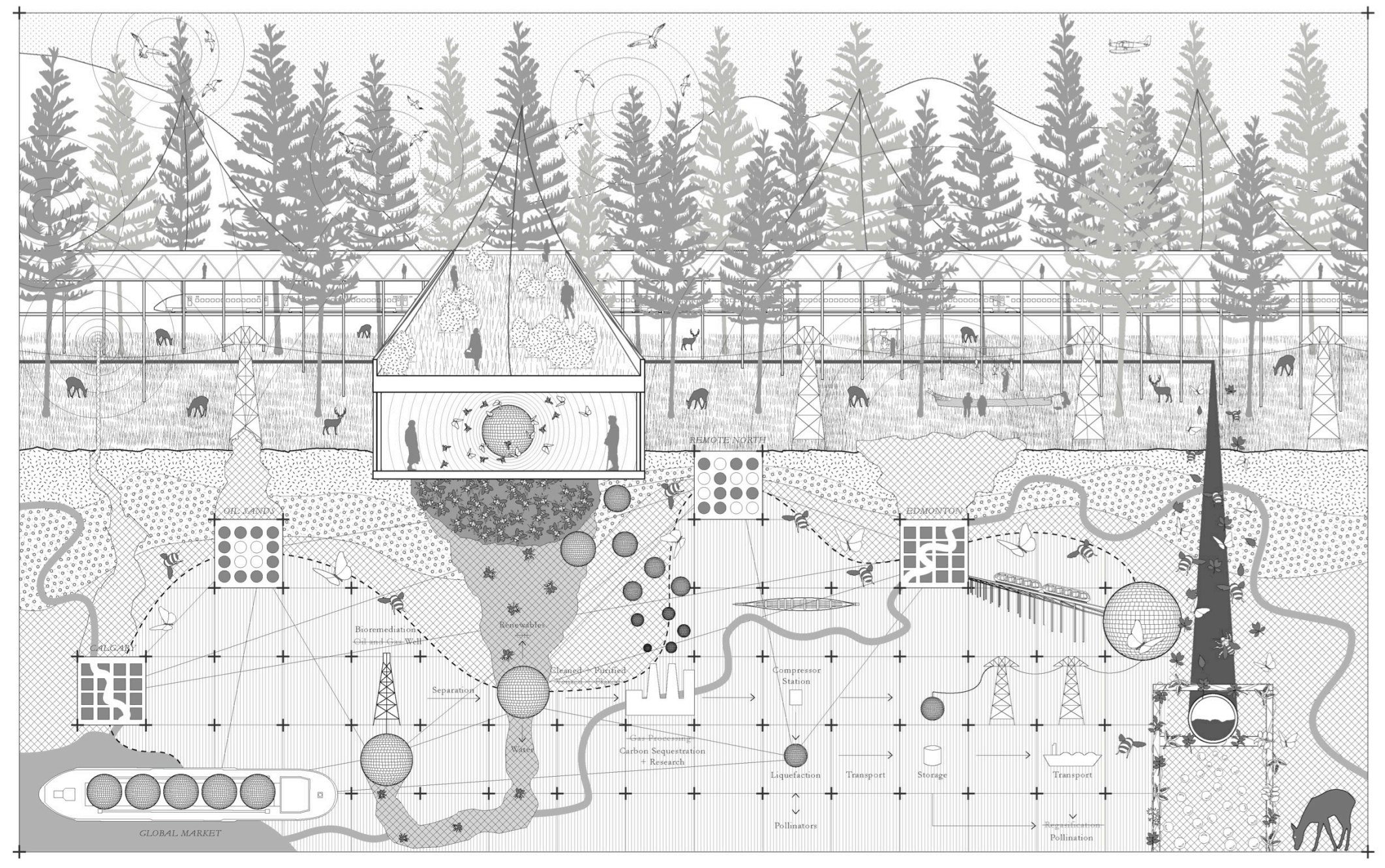




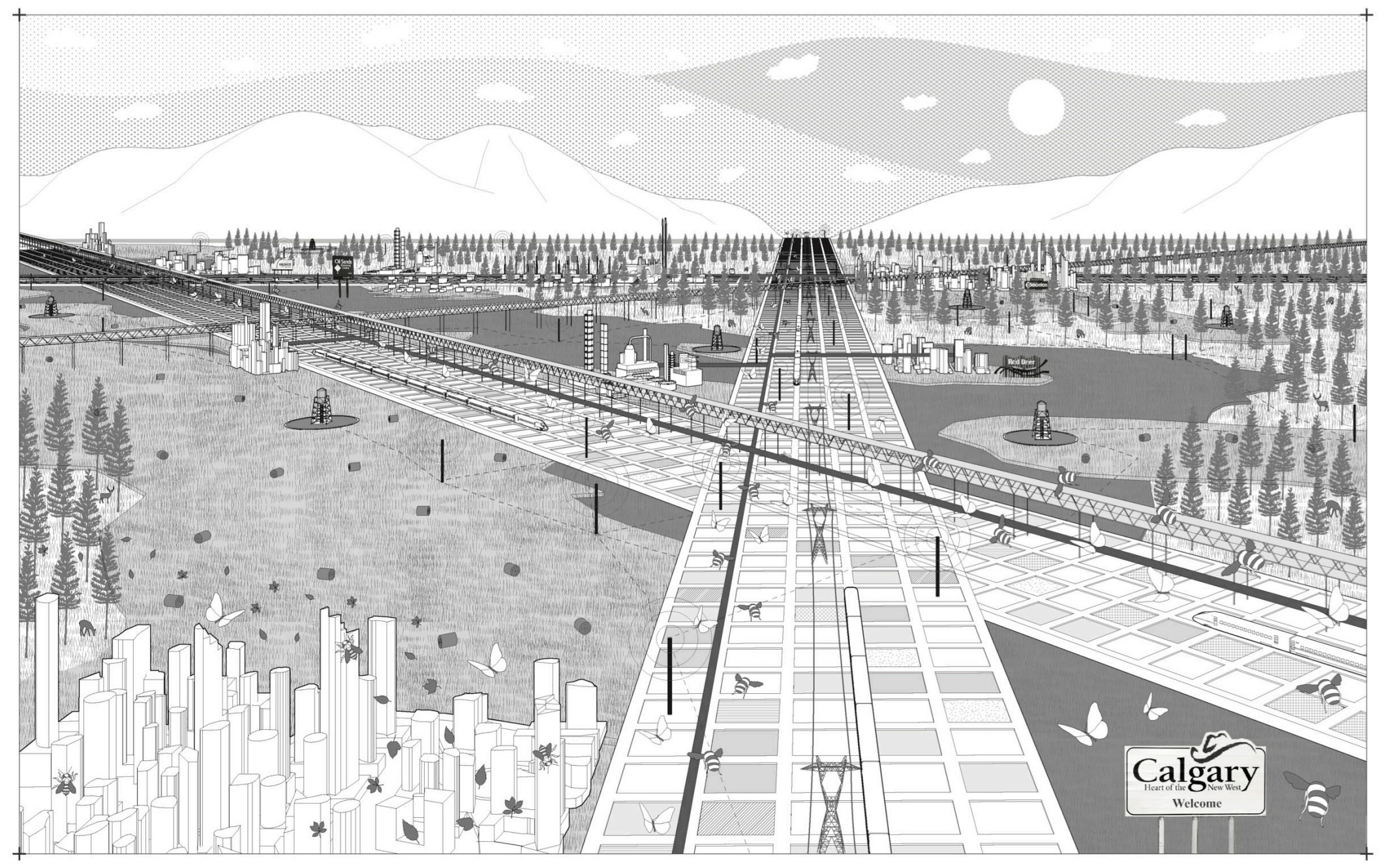




\section{Images}

Image 1:

Jesse Martyn, Earth, Digital Media, 2020.

Image 2:

Jesse Martyn, [Ex]traction, Digital Media, 2020

Image 3:

Jesse Martyn, The New Frontier, Digital Media, 2020.

\section{Endnotes}

1 Fumihiko Maki, Investigations in Collective Form, (Washington: School of Architecture, Washington University, 1964), 3. 